
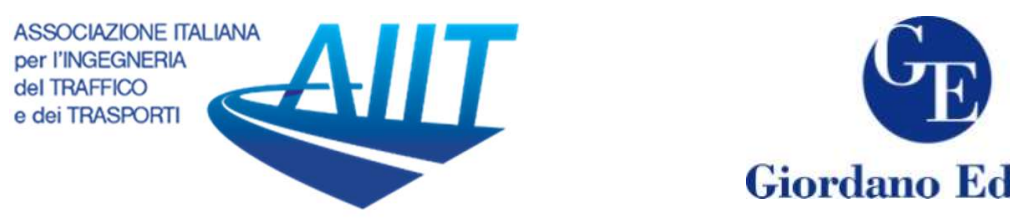

Giordano Editore

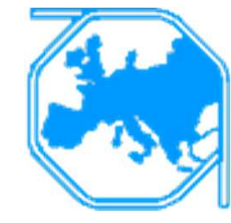

\title{
New Scenarios for Safe Mobility in Urban Areas Giulio Maternini ${ }^{1 *}$, Michela Tiboni ${ }^{1}$, MaurizioTira ${ }^{1}$
}

\author{
${ }^{1}$ Department of Civil, Environmental, Architectural Engineering and Mathematics, University of Brescia, \\ Italy.
}

\section{Editorial}

Since the mid-twentieth century, the International scientific community has highlighted the serious problem of road accidents. That problem became critical since the 1960 s due to the exponential increase of motorization in those years. Anyway, it was only at the end of the last century that the problem was addressed in a comprehensive and systematic way. Consequently, some effective measures have been implemented, and the first positive results were found. Nevertheless, in Europe, few countries have achieved the objectives given by the EU policies for the last three decades. For example, Italy has failed to reach the goal of halving the road victims in the decades 2001-2010 and 20112020 .

Therefore, we are still observing a high road victim rate per population and a poor awareness by civil society. It appears that road accidents have been accepted as a necessary factor in a developed society.

Investigating the places where accidents occur, the urban area is always very critical (about $75 \%$ of the casualties and seriously injured), confirming an almost stable number for many years. Furthermore, when looking at the type of user involved, it appears that the number of the most vulnerable users (i.e., pedestrians and cyclists) has not decreased in the last years. Therefore, we observe a greater safety of drivers and car passengers and a greater criticality in two-wheelers and non-motorized mobility. The figures are only partially justified by the numerous active safety devices that have been developed to assist car drivers (for example, the automatic braking system (ABS), preventing the vehicle from drifting, the cruise control and the electronic stability programme (ESB)) and passive safety facilities, such as the airbags. Those solutions have reduced the number of accidents and the severity of injuries, especially on ex-urban roads and motorways. In the urban environment, the efficacy of such devices is reduced by the complexity of the system, where several activities and different road users share the same spaces. Therefore, an unacceptable level of accidents and victims are still detected.

The $\mathrm{XXV}^{\circ}$ edition of Living and Walking in Cities Conference focused on the challenge of defining new scenarios for safe mobility in urban areas, according to the pillars of the EU strategy to promote safer, resilient, and innovative cities.

\footnotetext{
*Corresponding author: Giulio Maternini (giulio.maternini@unibs.it)
} 
The Special Issue "New Scenarios for Safe Mobility in Urban Areas" of the European Transport Journal contains a collection of twelve extended papers from the International Conference, a bi-annual appointment aiming to gather researchers, experts, administrators, and practitioners, to offer a platform for discussion about mobility and quality life in urban areas, with a specific focus on vulnerable road users' safety. The aim is to exchange ideas, theories, methodologies, empirical insight, experiences, and techniques about policy issues, best practices, and research findings.

The authors of the twelve extended articles revised the conference papers and added $30 \%$ new materials following the usual practice so that extended articles went through a new peer-review process.

Those articles can be divided into three main themes: mobility planning ( 7 papers), safety in intersections ( 3 contributions) and safety of vulnerable users ( 2 contributions).

The topic of mobility planning deals with several aspects, such as the location of services, the geometry of the roads, the operational speed of vehicles and the spaces for non-motorized mobility and urban micro-mobility. They address the topic of the 15minutes city, the environmental sustainability of the transportation systems, etc. Russo, Pellicanò, Liritano, Petrungaro \& Trecozzi in the paper "From global goals to local development: the role of regional plan for sustainable urban mobility" analyze the intervention proposed by the Regional Transport Plan of the Calabria Region (Italy), aimed to contain polluting emissions and transport costs, to promote safe mobility and to improve accessibility. The sub-topic of the 15-minutes city was deepened from three contributions. Gaglione, Gargiulo, Zucaro \& Cottrill in the paper "15-minute neighbourhood accessibility: a comparison between Naples and London" provide a methodology aimed at defining 15-minute neighbourhoods by adopting a systemic approach and tested it on some suburbs located in the cities of Naples and London. Similarly, Caselli, Carra, Rossetti \& Zazzi in the paper "From urban planning techniques to 15-minute neighbourhoods. A theoretical framework and GIS-based analysis of pedestrian accessibility to public services" assess the 15-minute neighbourhoods through a GIS-based model to evaluate pedestrian accessibility to urban services and spaces. The method was then applied to measure the current performance of an existing neighbourhood in Parma. Likewise, Pinto \& Akhavan in the paper "Scenarios for a Post-Pandemic City: urban planning strategies and challenges of making "Milan 15-minutes city" provide an overview of this concept and its application in the case of Milan during the second phase of the Covid-19 pandemic. Carpentieri, Papa \& Guida (Urban Planning for Transit-Oriented Development: An application in the Naples metropolitan area) provide an innovative methodology to assess areas around transit nodes and supports decision-makers in identifying critical assets for investment in the transit-oriented development planning strategy. Moreover, Carboni, Pirra, Costa \& Kalakou in the paper "Gender perceptions of active mobility: Insights from three European cities" indagate how the perception towards active mobility modes changes according to gender. They showed how women are more worried about safety, as they do not appreciate sharing spaces with motor vehicles. Finally, Fistola, Gallo \& La Rocca in the paper "Cities between smartness and emergencies: exploring the role of e-scooter in the transition era" address the emerging theme of the urban micro-mobility, focusing 
on the analysis of the spread of e-scooters in Italy, relating both to the pandemic and to the post-emergency phase.

The theme of safety at the intersection is a topical issue in the urban context, as the existing nodes has been frequently designed, mainly focusing on the motorized users only. Particularly, Gruden, Otković \& Šraml in the paper "Pedestrian safety at roundabouts: a comparison of the behavior in Italy and Slovenia" analyze the pedestrian safety at roundabouts in two Countries, Italy and Slovenia. They highlight the need to find solutions from both the infrastructural side and pedestrian safety awareness. Similarly, Distefano, Leonardi \& Pulvirenti in the paper "Experimental analysis of pedestrian behavior at different configurations of crosswalks at roundabout legs" investigate the pedestrian behaviour when crossing the legs of urban roundabouts by using video recordings. To encourage legal pedestrian behaviour, the findings suggested the implementation of refuge islands at the legs of roundabouts. Moreover, Kopsacheilis, Fourkiotis, Kazaklari \& Politis in the paper "Capacity of deep learning algorithms on modelling pedestrian behavior at crossings with countdown signal timer installations" examine the capacity of different techniques in the modelling of pedestrian crossing behaviour in an urban signalized intersection with a countdown signal timer. The chosen Multinomial Logistic Regression and Deep Learning models showed a good fit in the prediction of pedestrian conduct.

Finally, the theme of vulnerable users' safety has been addressed by Gitelman \& Korchatov in the paper "Exploring safety-related behaviours of e-cyclists on urban streets; an observational study". They explore the behaviour of e-cyclists conducting observational surveys in Israeli cities. They highlighted the need for improved cycling infrastructure, with separation from sidewalks and roadways. Besides, Pajković \& Grdinić-Rakonjac in the paper "Road users' attitudes and perception on selected road safety issues - age-related comparison" explore the behaviour of the young drivers, which are considered among the most vulnerable road users. Indeed, while they represent only a small percentage of licensed drivers, they are proportionally more involved in fatal and injury crashes than older ones. The results show that the perception of the overall safety situation differs in different age groups.

\section{Acknowledgements}

The chairs and organizing committee of LWC 2021 express their gratitude to the scientific committee, the contributors for their papers and presentations, the session chairs, and the reviewers for their efforts in making the conference successful and making the publication of this special issue possible. 